\title{
Evaluation of Percepcion of Red Color APPLIED TO KOFFKA EFFECT
}

\author{
MatiJevic, M.; MrvaC, N.; Milkovic, M. \& Vusic, D.
}

Abstract: Optical illusions are false perceptual phenomena, which often can be confusing. One of the fascinating optical illusion was made by Kurt Koffka. The effect consists of a grey ring located at the two bases of different shades of gray where the ring appears to be homogeneous. If the ring is slightly divided into two parts, the perception of the individual alone will greatly affect the background so that the part of the ring, which is underlying darker perceived brighter than the one that is on the lighter background. The visual perceptionis experienced differently in a comparison one against the other within our visual field - it is a perceptual, not the physical effect. In order to research that effect, research is focused on monitoring perceptual experience of stimulaneous contrast between ring and background of the ring which is coloured red.

Key words: Koffka, perception, contrast, colour
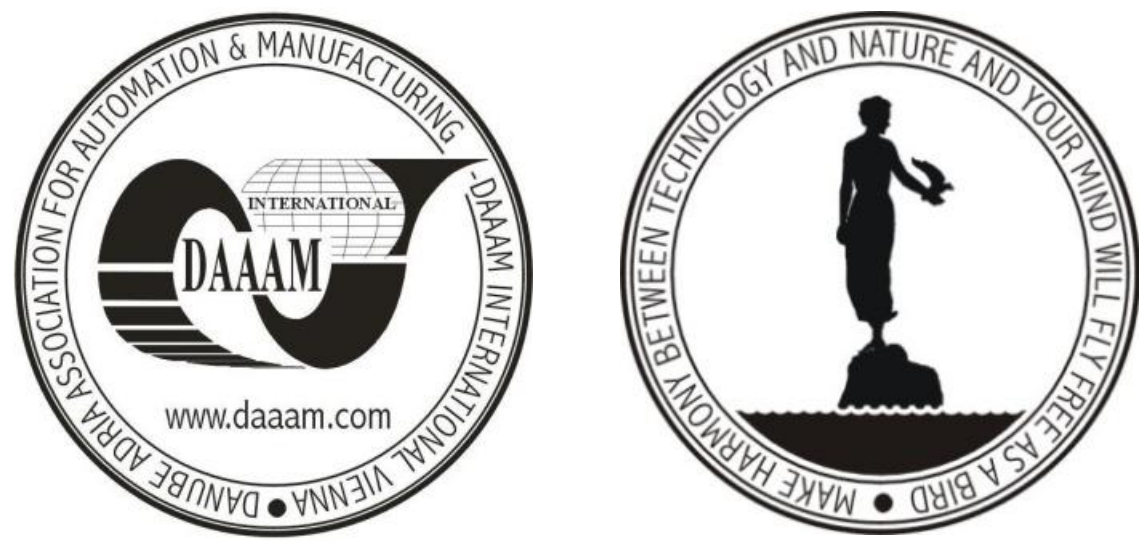

Authors' data: Matijevic, M[ile]*; Prof. Mrvac, N[ikola]*; Dr. Milkovic, M[arin]**, Vusic, D[amir]**,*Faculty of Graphic Arts, University of Zagreb, Getaldiceva 2, 10000, Zagreb, Croatia, **University of Applied Sciences, J. Krizanica 33, 42000, Varazdin, Croatia, mile.matijevic@grf.hr, nikola.mrvac@grf.hr, dekan@velv.hr,damir.vusic@velv.hr

This Publication has to be referred as: Matijevic, M[ile]; Mrvac, N[ikola]; Milkovic, M[arin] \& Vusic, D[amir] (2010). Evaluation of Percepcion of Red Color Applied to Koffka Effect, Chapter 26 in DAAAM International Scientific Book 2010, pp. 259-270, B. Katalinic (Ed.), Published by DAAAM International, ISBN 978-3901509-74-2, ISSN 1726-9687, Vienna, Austria

DOI: $10.2507 /$ daaam.scibook.2010.26 


\section{Introduction}

The very process of perception is what the human eye sees, while the base of perception isthe organization of data from the senses. In the case of visual perception, some people can actually see the perceptual shift in their Mind's Eye (Wettlaufer, 2003). Attention and adaptation aremechanisms that optimize visual effect. (Pestilli et al., 2007). Contrast also has a large influence on colour perception. It describes the influence of one color on color perception in the neighboring areas (Shepherd, 1999).

Stimulus light contrast is one of the oldest illusions of perception. Stimulus contrast isthe psychophysical visual effect that causes the shift of color appearance of a stimulus, and is caused by changing the background color. Despite more than a century of intensive research and study ofstimulus contrast, it is still confusing and poorly understood phenomenon (Kingdom, 2003; Shevell, 1978; Valberg, 1975; Whittle, 2003). It might even be said that stimulus contrast causes colour constancy (Hunt, 1979, 1991). Perceived colour depends on the spectral distribution of stimulus, its size, shape, structure, surface characteristics of the object from which the stimulus originates, complexity (in the case of image files) and the background and environment in which the stimulus is viewed, then on the angle of stimulus intrusion, adaptation state of observer's visual system, experience, attention etc. The important thing in the perception of colour is the understanding of the role of chromatic mechanisms in the area of perception (Huang et al., 2007).Stimulus contrast is a subjective psychological experience, and it proves the fact that the same physical stimulus (the same dominant wavelength) with different people caused different color experiences (Milkovic 2006; Kovacic et al., 2009). How that is perceptual experience it as such can not be physically measured.

\section{The Koffka effect}

Gestalt psychologist Kurt Koffka nearly seventy years ago has made a fascinating effect (Adelson, 2000; Koffka, 1967). Effect consists of three distinct, but related variants. The effect consists of a gray ring located at the two bases of different shades of gray. Such a ring appears to be homogeneous. On its perception influence the light and the dark background (Fig. 1 a). The second figure is a ring that is divided and is situated on two different surfaces so that a portion of the ring is perceived to be brighter, the part that is on the darker background and the second part of the ring, which is perceived to be darker, the part that is on the lighter background (Fig. $1 \mathrm{~b}$ ). The third image shows a ring which is divided into two parts, but unlike the figure $b$, where is a little torn here is one-half compared to the other vertically displaced (Fig. 1 c).Here on the perception of single part of the ring except of its base influence the second part of the ring and the substrate on which is the second part. Gestalt principle of continuity in visual perception could explain this effect (Adelson 2000). Each area is perceived as part of a whole and in perceptually ambiguous situations, some surfaces will perceive like it belongs to a whole, with which it best 
complements. How is experienced the brightness of a surface under the influence of the brightness of adjacent areas, a variety of perceptual organization can lead to different estimates of the brightness of the same area.

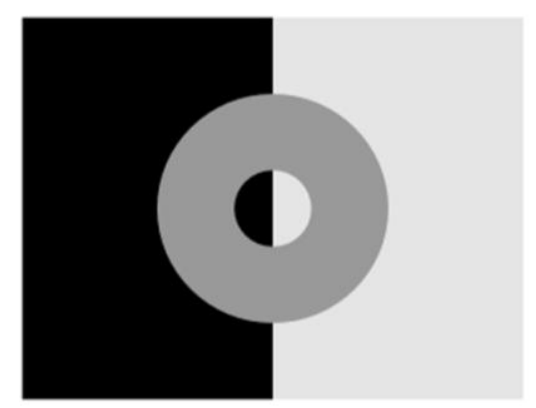

a
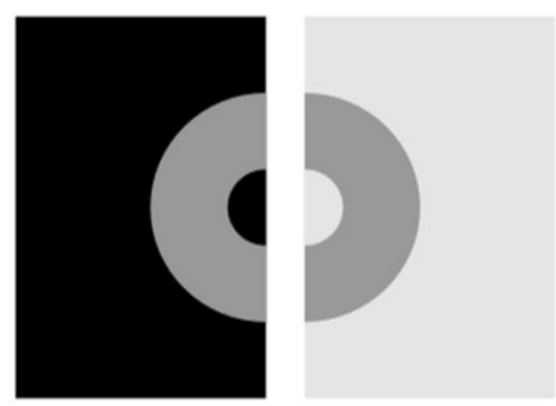

b

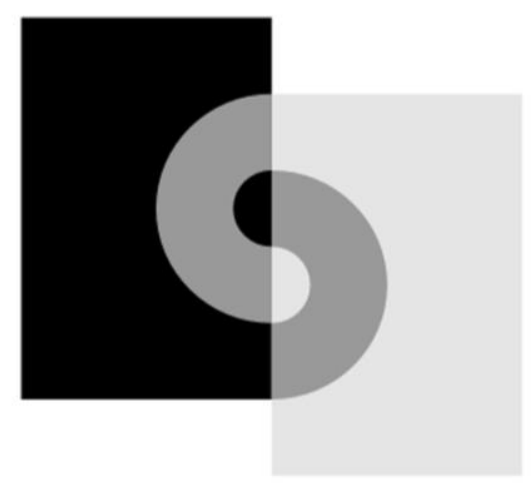

C

Fig. 1. The Koffka effect

Many studies include colour and its brightness (Beck, 1972; Wyszecki, 1986). Brightness is the feature of our perception intensity. It describes the amount of light. "Ownership" is of the object that is observed, and can be detected with a lot of light to see colour, unlike the other two characteristics. In the darkness people see with the sticks that do not distinguish colour, but using them the difference in brightness can be seen.

\section{Experimental part}

The paper applies the Koffka effect on the red colour of the RGB colour system. The study included 40 examinees, mixed population aged 20-24 years who had previously successfully meet the criteria of Ishihara test for the detection of potential defectionof vision. The Koffka model is separated into five separate units and for each unit separately a visual assessment is performed (Fig. 2, 3, 4). The testing was done on computers with a calibrated monitor in a manner that subjects should determine the colourtone of the part of the ring compared to the scale that was attached. Scale consisted of seven fields horizontally oriented, from darker to lighter. 
Matijevic, M.; Mrvac, N.; Milkovic, M. \& Vusic, D.: Evaluation of Percepcion of ...

The difference between each field was 5 values of RGB scale. For testing were madethree variations of the Koffka effect depending on the staining of the substrate and the ring itself.

In the first variation of the background staining was 64 RGB value (left side of the substrate), 127 RGB value (right side of substrate) and 191 RGB value (the ring)

In second variation staining of substrate was 127 RGB value (left side of the substrate), $191 \mathrm{RGB}$ value (right side of substrate) and 64 RGB value (the ring).

In the third variation of substrate staining was $191 \mathrm{RGB}$ value (left side of the substrate), 64 RGB value (right side of the substrate) and 127 RGB value (the ring) (Table 1).

The study was based on the perception of the ring, depending on its brightness and relative brightness of the background. Each participant had to determine the field in a scale that suited him identical by perception of the ring staining (Fig. 2), or part of the ring (Fig. 3, 4).

\begin{tabular}{|l|l|l|l|}
\cline { 2 - 4 } \multicolumn{1}{c|}{} & $\begin{array}{c}\text { The value } \\
\text { of the } \\
\text { colour }- \text { left } \\
\text { background }\end{array}$ & $\begin{array}{c}\text { The value } \\
\text { of the } \\
\text { colour }- \\
\text { right } \\
\text { background }\end{array}$ & $\begin{array}{c}\text { The value } \\
\text { of the } \\
\text { colour of } \\
\text { the ring }\end{array}$ \\
\hline Variation 1 & 64 & 127 & 191 \\
\hline Variation 2 & 127 & 191 & 64 \\
\hline Variation 3 & 191 & 64 & 127 \\
\hline
\end{tabular}

Tab. 1. Values of staining of the test patterns

Environmental conditions for visual evaluation were within the guidelines of ISO 12646 fromyear2004. Time for the valuation was not limited.

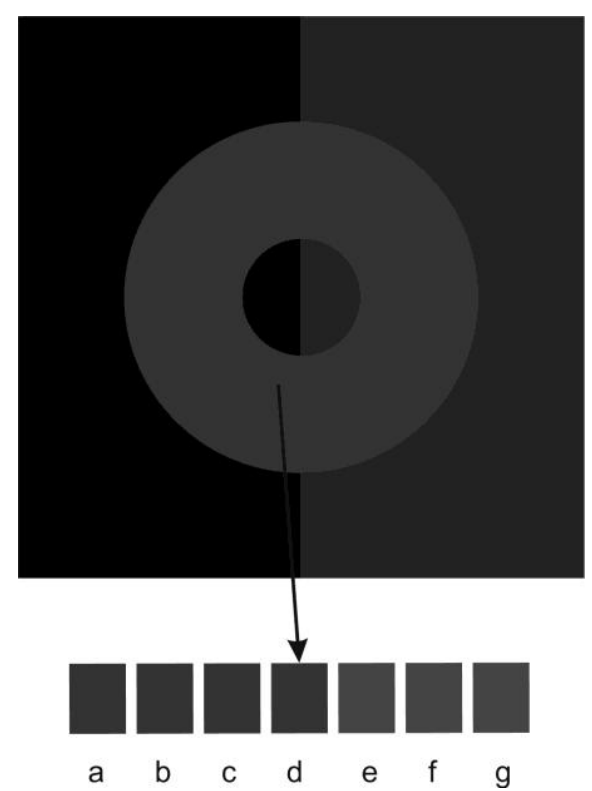

Fig. 2. Display priciple of visual evaluation of the hole ring 


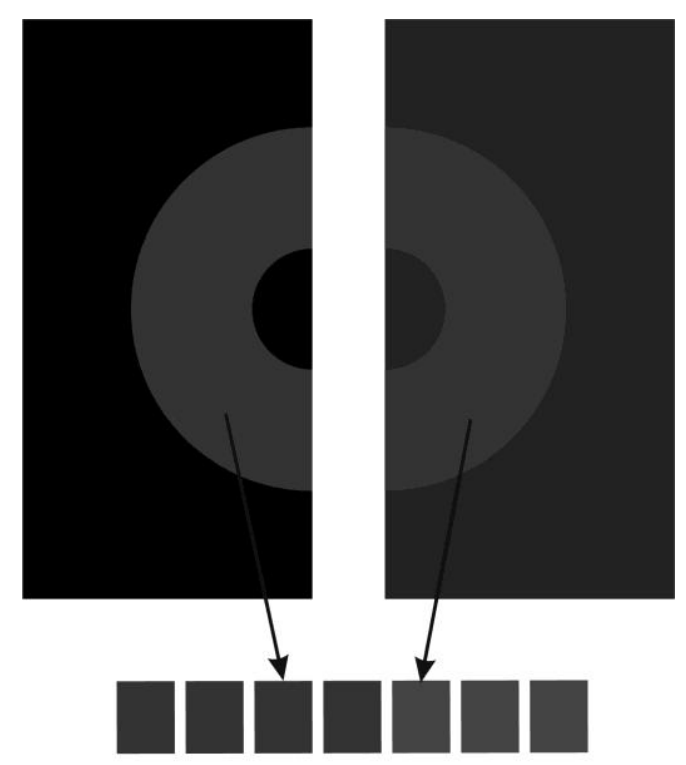

a $\quad b \quad c \quad d \quad$ e $f \quad g$

Fig. 3. Display priciple of visual evaluation left and right part of the ring

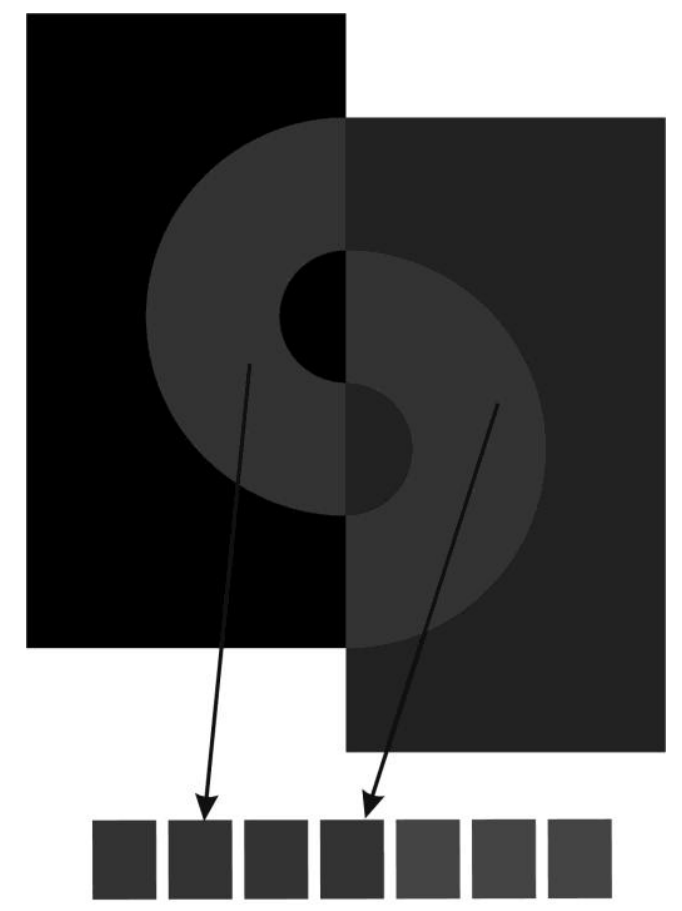

$\begin{array}{lllllll}a & b & c & d & e & f & \end{array}$

Fig. 4. Display priciple of visual evaluation left and right part of the ring

\section{Research results}

In Figures 4-11 the results obtained by visual perception of the Koffka effect on which is applied the red color from the RGB system are presented. In Figures $4-$ 6 the results obtained and compared with identical substrates related to the perception of the tests are presented (Fig. 2, 3, 4). 
Matijevic, M.; Mrvac, N.; Milkovic, M. \& Vusic, D.: Evaluation of Percepcion of ...

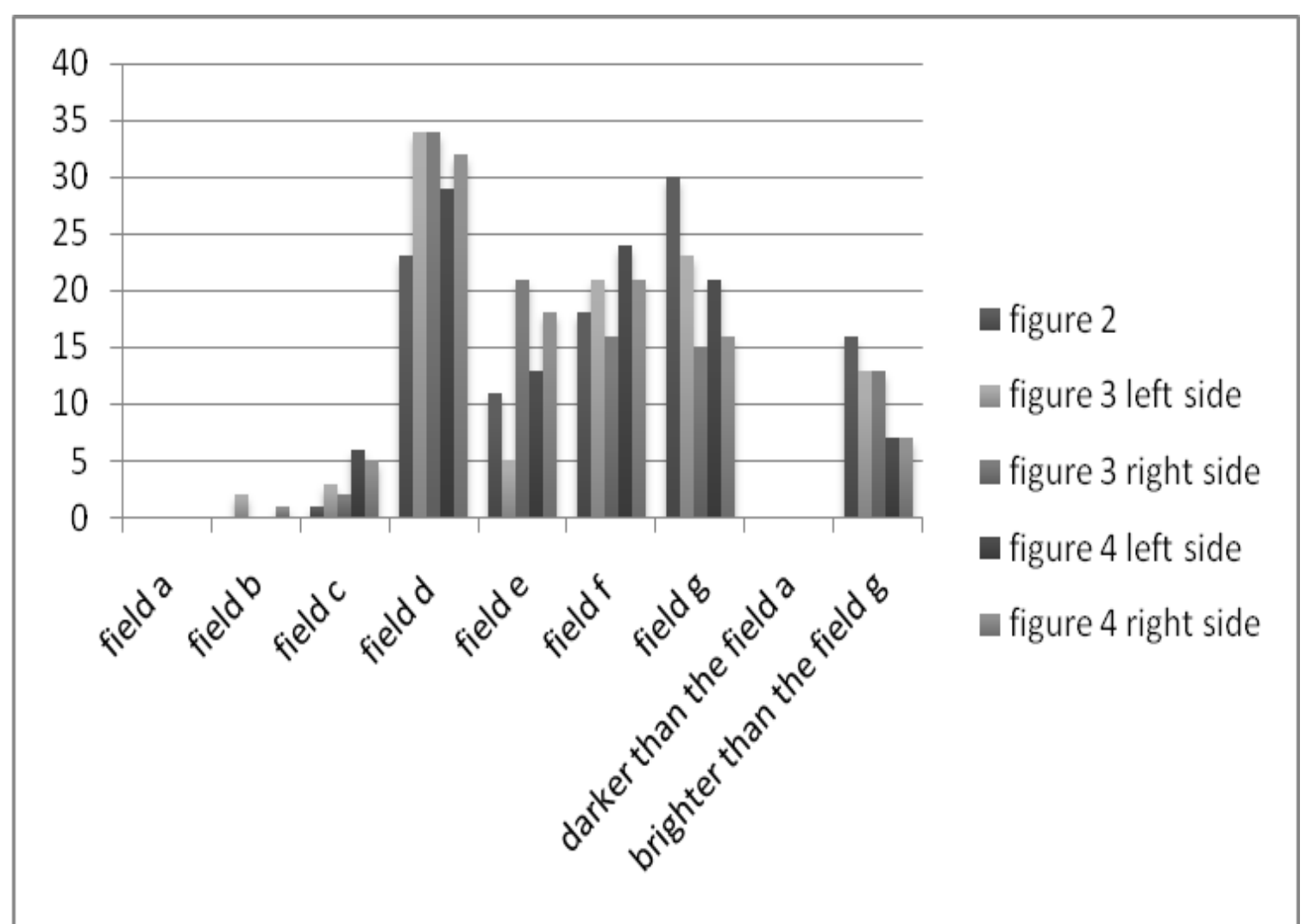

Fig. 4. How the precentages of responses depending on the field in the scale of the Koffka effect in the first variation

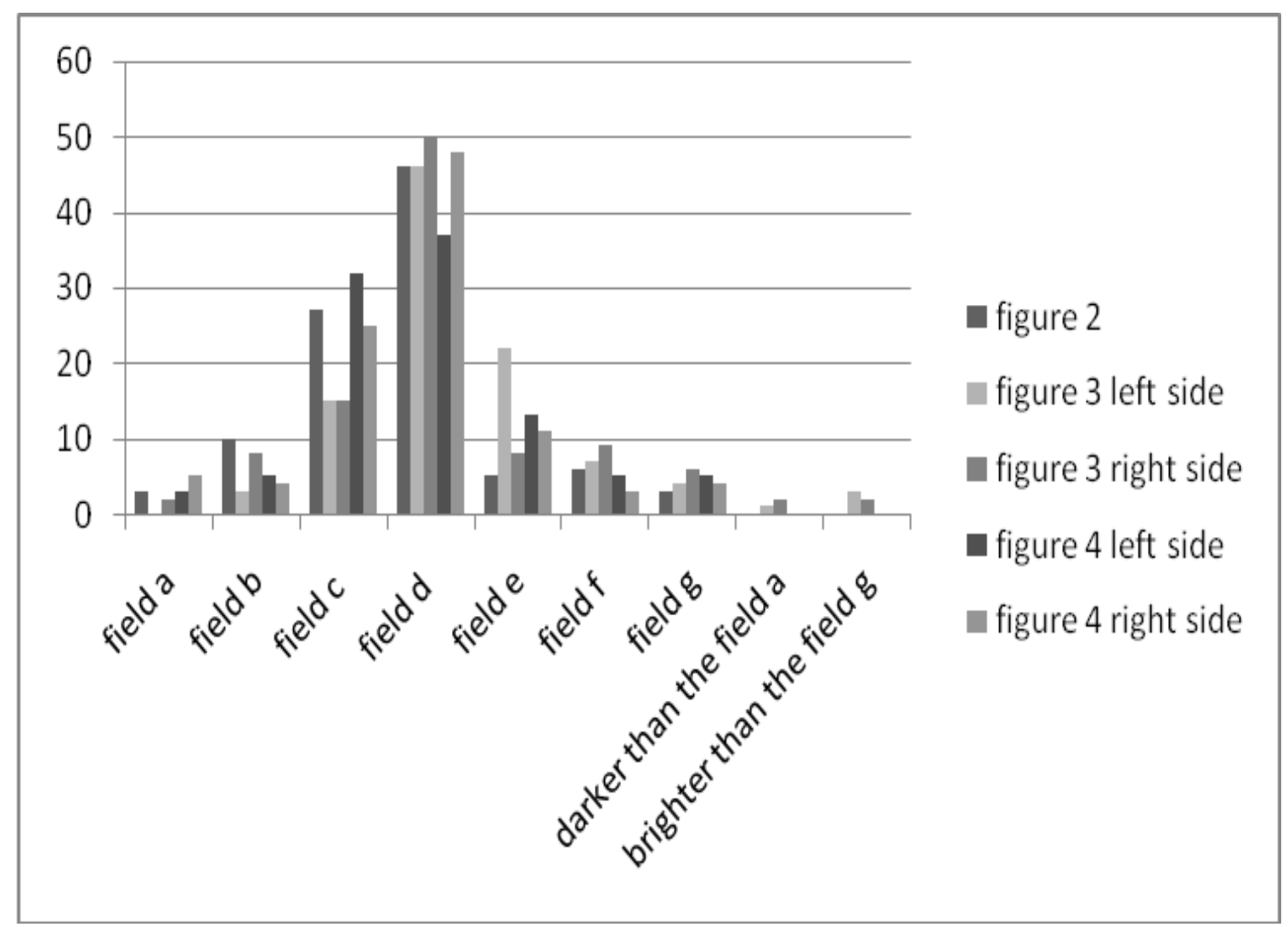

Fig. 5. How the precentages of responses depending on the field in the scale of the Koffka effect in the second variation 


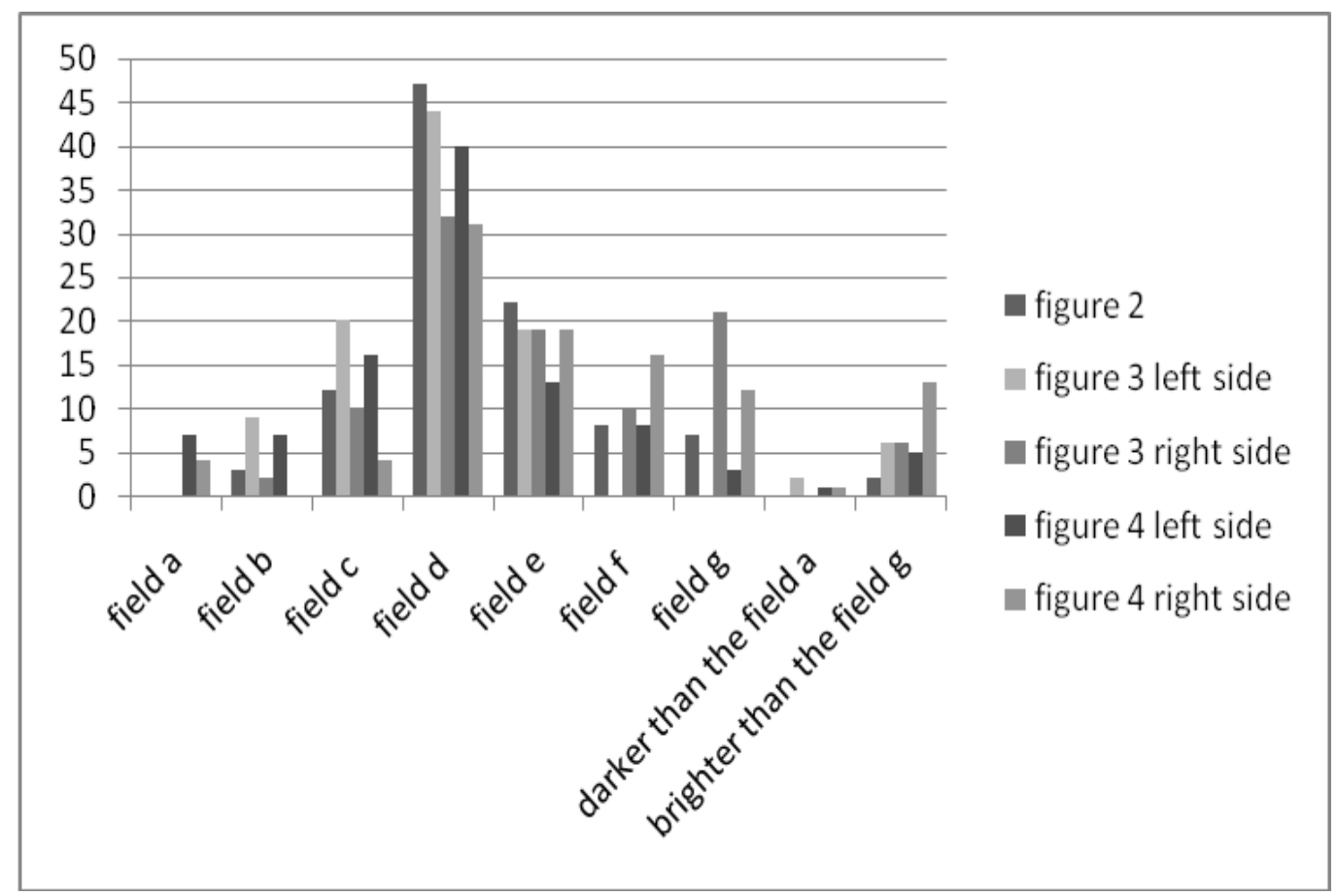

Fig. 6. How the precentages of responses depending on the field in the scale of the Koffka effect in the third variation

In the Figures7 - 11 the results obtained by the perception of different staining of background and the ring are presented and compared. In the graph legend the first number represents the staining of left background, second number right background and third number staining of the ring.

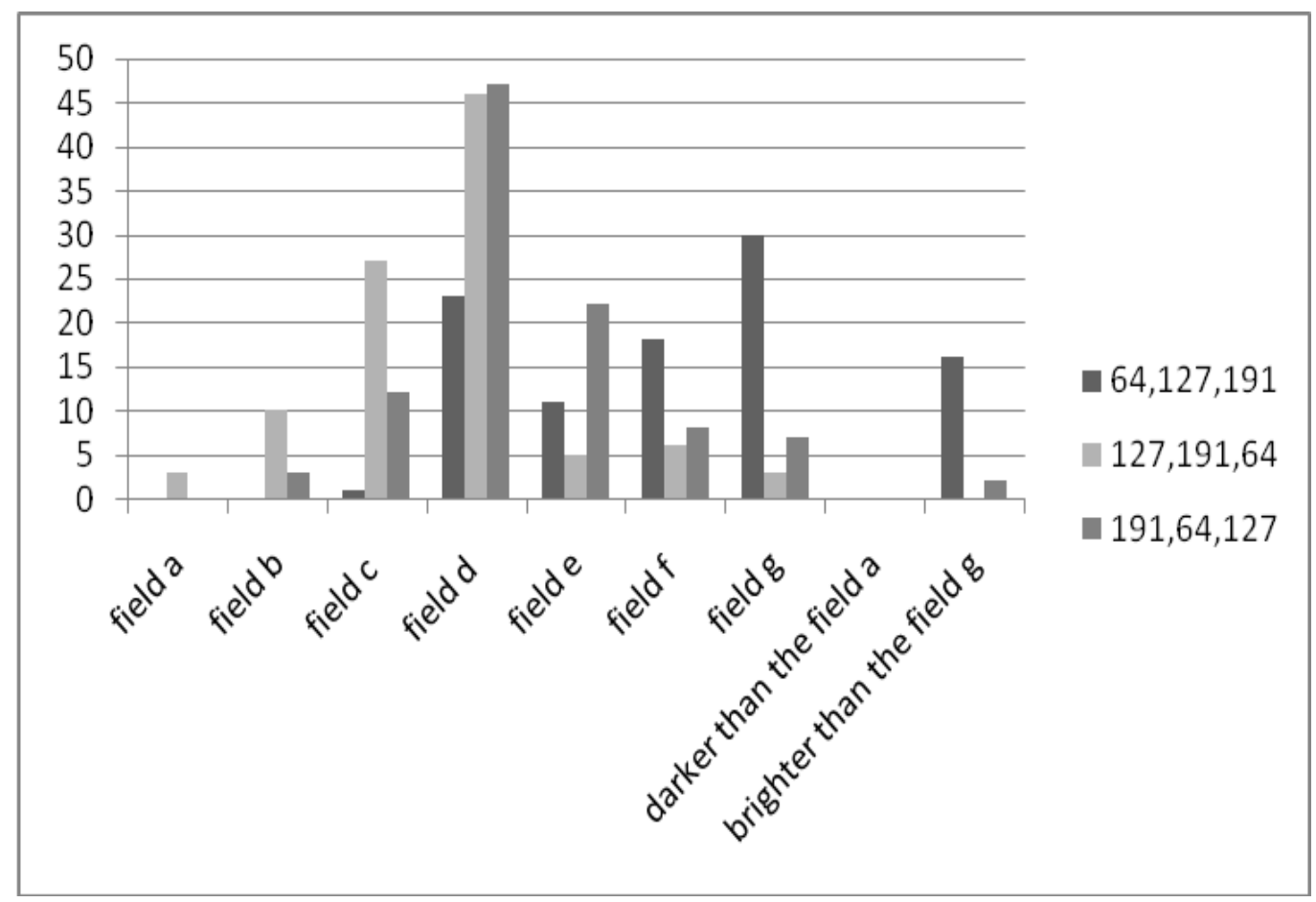

Fig. 7. The relationship betwen percentages of responses depending on the field in a scale comparing all three variations of staining of the substrate and the ring in the koffka effect test shown in figure 2 
Matijevic, M.; Mrvac, N.; Milkovic, M. \& Vusic, D.: Evaluation of Percepcion of ...

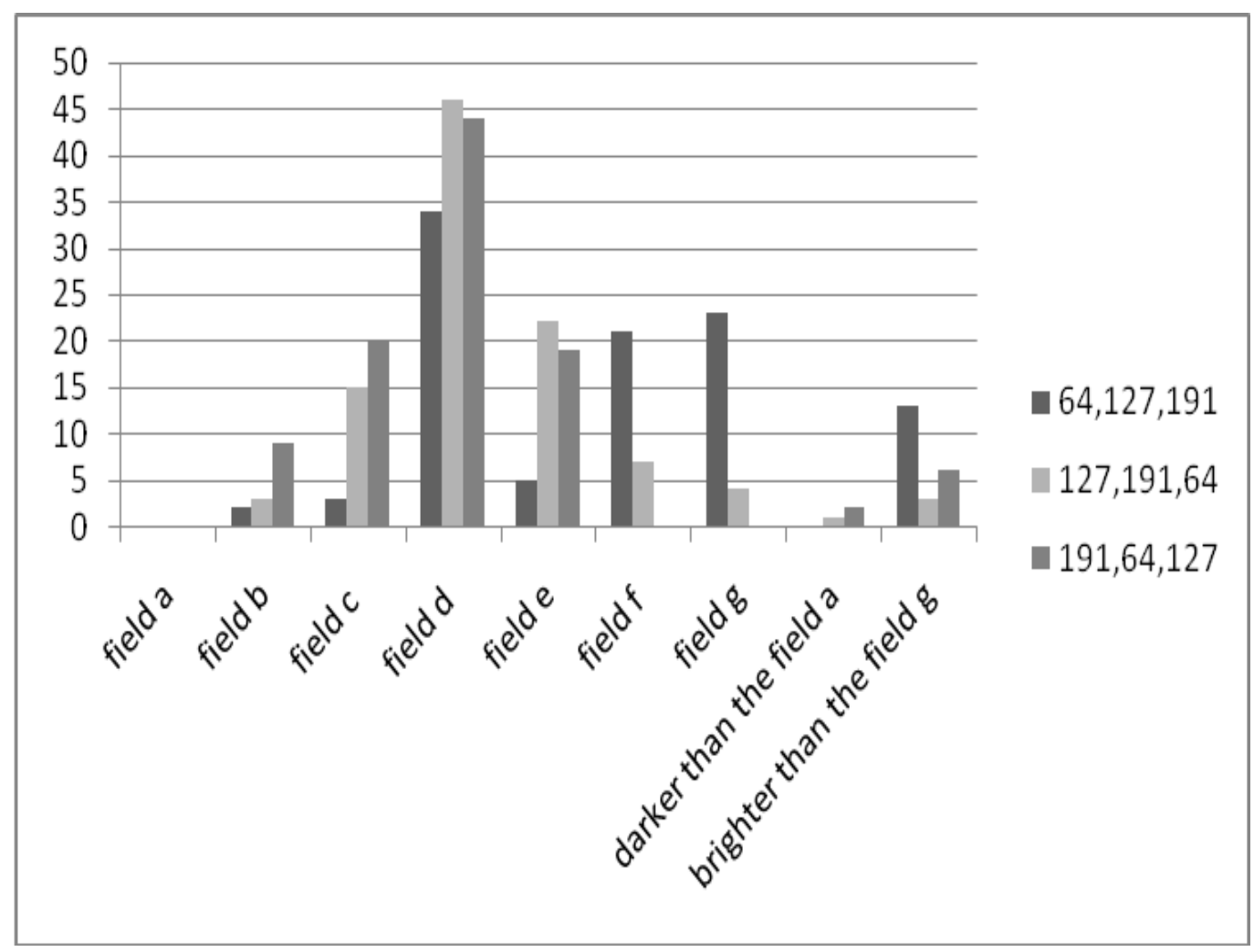

Fig. 8. The relationship betwen percentages of responses depending on the field in a scale comparing all three variations of staining of the substrate and the ring in the koffka effect test shown in figure 3 (left side)

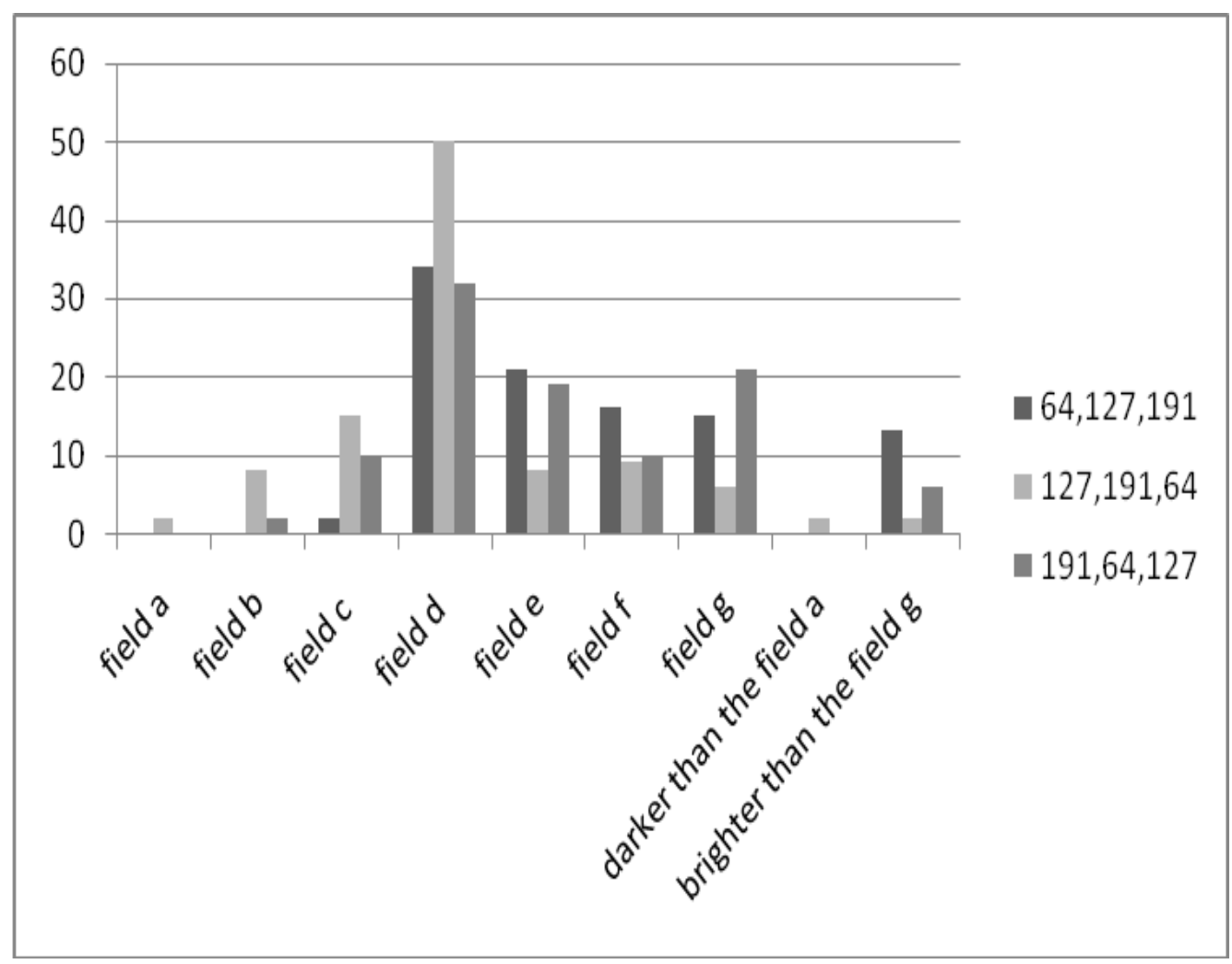

Fig. 9. The relationship betwen percentages of responses depending on the field in a scale comparing all three variations of staining of the substrate and the ring in the koffka effect test shown in figure 3 (right side) 


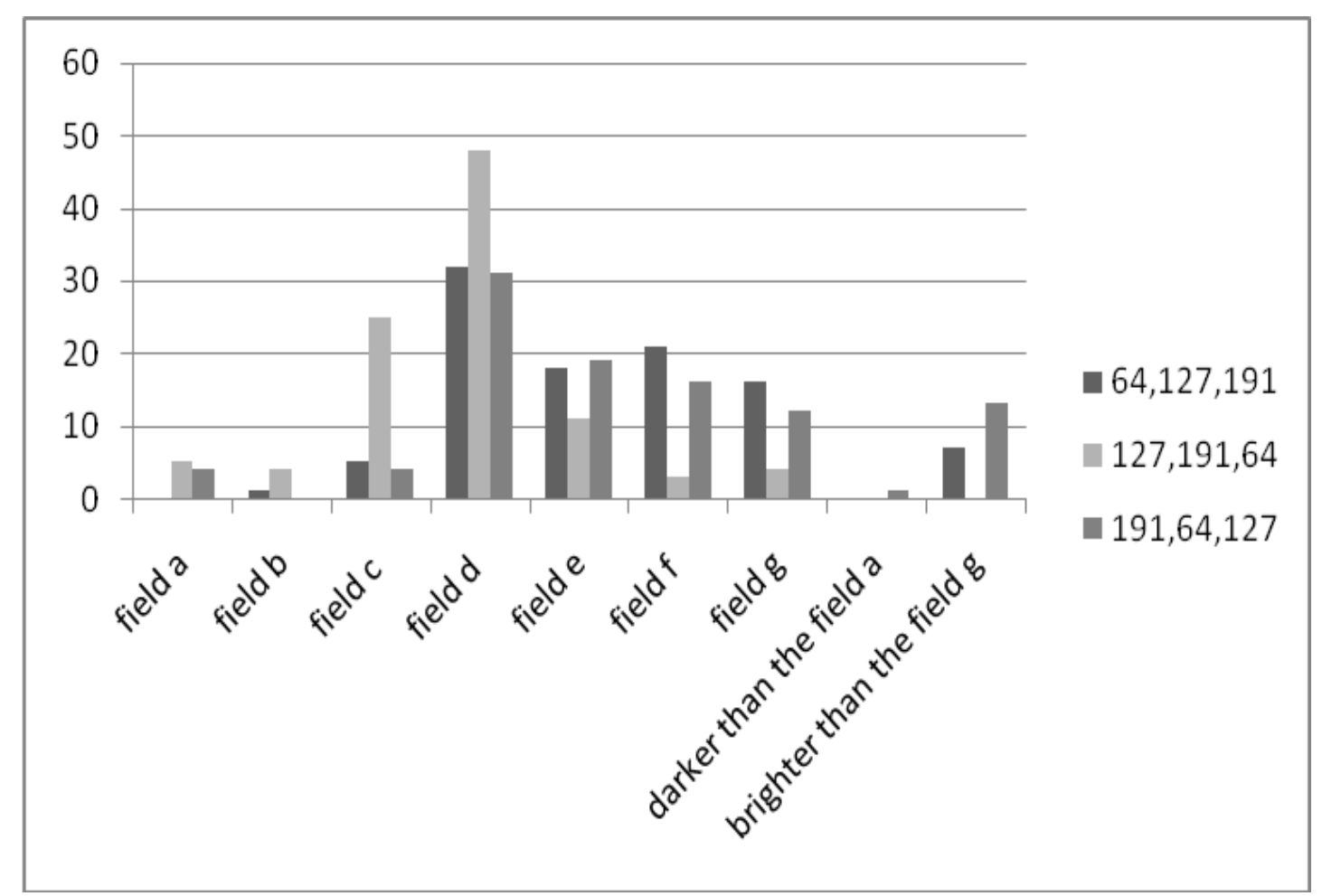

Fig. 10. The relationship betwen percentages of responses depending on the field in a scale comparing all three variations of staining of the substrate and the ring in the koffka effect test shown in figure 4 (left side)

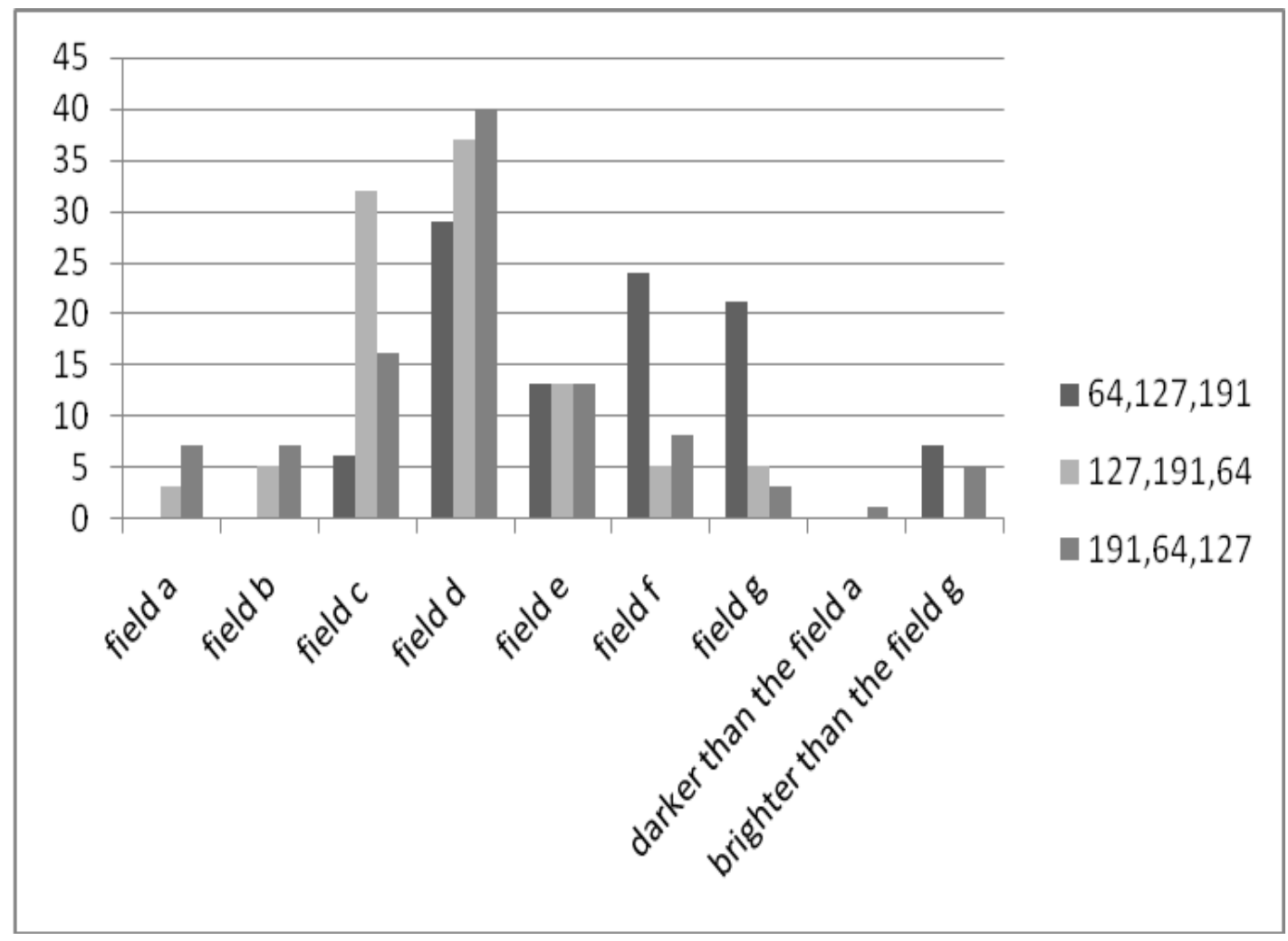

Fig. 11. The relationship betwen percentages of responses depending on the field in a scale comparing all three variations of staining of the substrate and the ring in the koffka effect test shown in figure 4 (right side) 
The first Koffka effect test where the values of staining substrate have RGB value 64 (left side), 127 (right side) and 191 (ring) the average of correct answers for all five tests were $30.4 \%$ (Fig. 4). The results show that for the field e we have a large range of responses of 5\% (Fig. 3 left side) up to 21\% (Fig. 3 right side).Equally large number of respondents decided for the $\mathrm{g}$ field 15\% (Fig. 3 right side), 16\% (Fig. 4 right side), 21\% (Fig. 4 left side), 23\% (Fig. 3 left side), and $30 \%$ (Fig. 2).

In the second Koffka effect test (Fig. 5) where the values of substrate staining have RGB value 127 (left side), 191 (right side) and 64 (ring) the average of correct answers for all five tests amounts $45.4 \%$. For darker fields, or for $\mathrm{a}, \mathrm{b}$ and $\mathrm{c}$ fields, decided more participantsthan for $\mathrm{e}, \mathrm{f}$ and $\mathrm{g}$ fields.

In the third Koffka effect test (Fig. 6) where the value of staining substrate have RGB value 191 (left side), 64 (right side) and 127 (ring) the average of correct answers for all five tests was $38.8 \%$. Correct answers have a very wide range which can be seen from the graph, from 31\% (Fig. 4 right side), 32\% (Fig. 3 right side), $40 \%$ (Fig. 4 left side), 44\% (Fig. 3 left side), to $47 \%$ (Fig. 2). Wide ranges have field $\mathrm{f}(0 \%-16 \%)$ and the field $\mathrm{g}(0 \%-21 \%)$ that were brighter than the color of the ring.

If the results for all three tests are compared it is visible that the highest perception is of the red ring with RGB value 127 and that amouts $47 \%$ (Fig. 7). The smallest perception is of the RGB value 191 and that amount sonly $23 \%$.

It is also visible that the ring is with RGB value 191 perceived brighter.If the results for all three tests are compared(Fig. 3 left side) best is perceived red ring with RGB values of 64 which amounts $46 \%$ and the lowest is perceived red ring with RGB value 191 which amounts $34 \%$ (Fig. 8).

It is interesting to note also that the perception of the ring with the RGB value 191 is brighter and amouts even $21 \%$ of respondents that perceived it lighter and for RGB values of 10 of the ring colour scale, and $23 \%$ as the value of field $\mathrm{g}$, which is lighter 15 RGB valued of the scale.

If the results for all three tests are compared(Fig. 3 right side) best is perceived red ring RGB values of 64 which amounts 50\% (Fig. 9). For the value 191 RGB of the ring it amounts $34 \%$, while for the value $127 \mathrm{RGB}$ of the ring it is only $32 \%$. It is interesting to note that $21 \%$ of respondents perceived the red ring with value 127 RGB brighter than the actual staining for 15 values of RGB scale.

If the results for all three tests are compared (Fig. 4 left side) best is perceived red ring with 127 RGB value which amounts $40 \%$ (Fig. 10). For the value 64 RGB of the ring it is $37 \%$. At least correct answers were if the ring had 191 RGB values which amounts only $29 \%$.

It is interesting to note that at the values of 191 RGB field perception of the ring is brighter $-24 \%$ as the tone of the field $\mathrm{f}$ which is lighter for 15 values on the RGB scale and $21 \%$ as the tone of the field $\mathrm{g}$ which is $20 \mathrm{RGB}$ values lighter from the staining of the ring.If the results for all three tests are compared(Fig. 4 right side) the best is perceived red ring with RGB values of 64 which amounts $48 \%$ (Fig. 11).

For the ring value of $191 \mathrm{RGB}$ it amounts $32 \%$. At least correct answers were if the ring with 127 RGB values only $31 \%$. It is interesting to note that the values of 
127 RGB field perception of the ring is brighter for $18 \%$ as the tone of field e or 10 RGB scales values of $21 \%$ as the tone of the field $\mathrm{f}$, which is 15 values of the RGB scale.

If we compare all three tests completely with each other the best perception we have by test number two, while the weakest by test number one, although they have almost identical values of color contrast between the background and the ring that we perceived. It is interesting to note that the test number three has the smallest difference in color contrast between the background and the ring but the perception is better than the test number one.

\section{Conclusion}

The Gestalt psychologists (Köhler 1992; Koffka 1967) were good in describing many illusions that are simple in presentation but they are complicated in the perception. The mere demonstration of Koffka effect shows how the spatial configurations may affect the ease of perception, and if in this effect another information is included, which is the color that is received through the sense of sight, then its description or specification belongs in the field of spectro photometry and spectro radiometry which also do not describe the way in which the color is perceived by eyes.

Specifically it would be that the dominant wavelength is perceived as the color under certain defined conditions of viewing. When viewing the test samples, there are other variables that are observed which also affect the perception of color and their interrelatedness with the goal of a more accurate description of colors

The aim was to investigate the strength of the background impact. The focus was to investigate the strength stimulus contrast and its impact on the perception ofbright tones and dark tones of the red colour as one of the primary additive colours.

Consequently the obtained results indicate that respondents perceive different stimulus contrast, and that its size varies depending on colour, saturation of the substrate and the contrast. Also surrounding colours affect the way some colours are seen.

The result of perception is except with the image based on the work of our visual system which is sometimes wrong, and just above the illusion visual perception is not perfect.

To obtain a more general picture about the possibility of defining stimulus contrast the expansion of this research on other psychophysical effects is anticipated.

\section{References}

Adelson, E. H. (2000). Lightness perception and lightness illusion, In: The New Cognitive Neurosciences 2nd edition, Gazzaniga, M. S. (Ed.), MIT Press, pp339-351, ISBN 978-0262071956, Cambridge, MA 
Matijevic, M.; Mrvac, N.; Milkovic, M. \& Vusic, D.: Evaluation of Percepcion of ...

Beck, J. (1972). Surface Color Perception, Cornell University Press, ISBN 9780801407048, Ithaca

Huang, Pi-Chun.; Mullen K. T. \& Hess R. F. (2007). Collinear facilitation in color vision, Journal of Vision, Vol. 7, No. 11, 2007, pp 1-14, ISSN 15347362

Hunt, R. W. G. (1979). Measures of colour appearance in colour reproduction. Color Research and Application, Vol. 4, pp 39-43, ISSN 1520-6378

Hunt, R. W. G. (1991). Revised colour-appearance model for related and unrelated colours. Color Research and Application, Vol. 16, pp 146-165, ISSN 15206378

Kingdom, F. A. (2003). Levels of brightness perception. Harris, L. \& Jenkin, M. (Ed.), Levels of perception, pp 23-46. Springer-Verlag. ISBN 978-0387955254, Berlin

Koffka, K.(1967).Principles of Gestalt Psychology, Hardcourt, ISBN 9780156744607, San Diego

Köhler, W. (1992).Gestalt Psychology, Liveright Publishing Corporation, ISBN 9780871402189, New York

Kovacic, A.; Matijevic, M.; Mrvac, N. \& Milkovic, M. (2009). Evaluation of the influence of the background colour on the perception of the stimulus contrast.Ananals of DAAAM for 2009 \& Proceedings of the 20th International DAAAM symposium, Katalinic, B. (Ed.), pp.1239-1240,ISBN 978-3-90150970-4, Vienna, Austria, November 2009, DAAAM International Vienna 2009, Vienna

Milkovic, M. (2006). Evaluation of psychophysically determinated effects and methods of gamut redefinition, Dissertation, Faculty of Graphic Arts, Zagreb

Pestilli, F.; Viera, G.\& Carrasco, M. (2007). How do attention and adaptation affect contrast sensitivity?, Journal of Vision, Vol. 7, No. 7, 2007, pp 1-12, ISSN 15347362

Shepherd, A.J. (1999). Remodelling colour contrast: implications for visual processing and colour representation, Vision Research, Vol. 39, pp 1329-1345, ISSN 0042-6989

Shevell, S. K. (1978). The dual role of chromatic backgrounds in color perception. Vision Research, Vol. 18, pp 1649-1661.ISSN 0042-6989

Valberg, A. (1975). Color induction. A study of lateral interactions in human vision. $\mathrm{Ph}$. D. thesis, Institute of Physics, University of Oslo.

Wettlaufer, A. K. (2003).In the mind's eye: the visual impulse in Diderot, Baudelaire and Ruskin, Rodopi, pp 257, ISBN 9042010355, Amsterdam

Whittle, P. (2003). Contrast colours. In: Colour perception: Mind and the physical world, Mausfeld. R. \& Heyer D. (Ed.), pp 115-138, Oxford University Press, ISBN 978-0198505006, New York

Wyszecki, G. (1986). Color appearance, In: Handbook of Perception and Human Performance:Sensory Processes and Perception, Boff, L. K.; Kaufman, L. \& Thomas, J. P. (Ed.), Vol. 1,pp 9.1-9.56, John Wiley \& Sons, ISBN 9780471885443, New York 